\title{
RECENT OBSERVATIONS OF THE BEAMS IN SS 433
}

\author{
R.C. VERMEULEN \\ Leiden Observatory \\ P.O. Box 9513 \\ 2300 RA Leiden \\ The Netherlands
}

\begin{abstract}
Preliminary results are presented of an intensive multifrequency observing campaign on SS 433 conducted in 1987. There were participants from all over the world; they brought all the observations together at the 1988 Dwingeloo workshop on SS 433. With a rapid sequence of VLBI maps, we have been able to follow the onset and evolution of a series of radio flares. We have also obtained a wealth of optical spectra allowing studies of the variability of the Doppler shifted lines on timescales of hours. There is little correlation between the activity in the radio regime, and that seen in the Doppler shifted lines.
\end{abstract}

\section{Introduction}

A decade has now elapsed since the discovery of the unusual nature of the binary system SS 433. An excellent review of the knowledge gathered during the first five years has been given by Margon (1984). The single most peculiar phenomenon in SS 433, the "moving lines" in its optical spectrum, was by that time thoroughly described in terms of the "kinematic model", which was first given in nearly its present form by Abell and Margon (1979). The kinematic model assumes only that matter is ejected from a component of SS 433 in two oppositely oriented collimated beams, and that the beam axis precesses. The physical conditions governing the ejection, collimation and propagation of the beams are not specified.

The kinematic model parameters were first determined from the optical moving lines, which show the radial velocity component of the beams. Later, radio maps, which show the shape and motion of the beams projected onto the plane of the sky, were made with the VLA by Hjellming and Johnston (e.g., 1985) and with VLBI (e.g., Vermeulen et al. (1987) and Romney et al. (1987)). These maps confirmed the kinematic model parameters and unambiguously fixed the direction of the precession axis in space; they also allowed an independent distance estimate to SS 433.

Much of the research into SS 433 was prompted by the hope that this system would prove to be a nearby, and therefore relatively easy to observe, version of the extragalactic jet sources associated with quasars. Indeed, SS 433 seems to have more in common with those extragalactic sources, discussed earlier in this Joint Discussion, than with the more typical galactic outflow sources treated in this session. For, just like the well-collimated jets in those large systems, the beams of SS 433 have an opening angle of only a few degrees (see Margon (1984)). The relativistic bulk speed, $0.26 c$, too, likens SS 433 more to quasars 
than to, say, Herbig-Haro objects. Again, just like in classical double radio sources, one can trace the beams of SS 433 over at least eight orders of magnitude in size. On the one hand, the beams in SS 433 are accelerated to terminal speed within $10^{12} \mathrm{~cm}$, as was deduced from EXOSAT spectroscopic observations by Watson et al. (1986). On the other hand, the radio shell of W50, which has a diameter of about $10^{20} \mathrm{~cm}$, has clearly undergone the influence of the beams of SS 433 (see for instance the radio map by Downes et al. (1981)).

\section{Overview of the 1987 Campaign}

In order to exploit the advantageous scales offered by SS 433 in both time variability and angular size, compared to active galaxies, we have organised an intensive monitoring campaign in the spring of 1987 . We concentrated on obtaining very frequent measurements in a limited (fourteen day) period at the end of May (JD 2446933 to JD 2446947), but the first measurements were made on JD 2446920 and the last on JD 2446957.

All participants in the campaign were invited to a workshop held on June 21 and 22 1988 in Dwingeloo, where the observations were presented and discussed. We will try to publish all our data as a series of articles in a single issue of a scientific journal.

The campaign was scheduled as close as possible to a time when the Doppler shifted lines reach their maximum separation; that is, when the angle between the beams of SS 433 and the line of sight is at its minimum. Differential effects between the approaching and the receding beam are then as large as possible. We wished to compare our observations to those made in an earlier, less extensive campaign in 1985 , when the beams were at right angles to the line of sight (see Vermeulen et al. (1987) and Vermeulen et al. (1989a)).

We aimed to obtain a sequence of high dynamic range VLBI maps, spaced so closely in time that any changes could be properly followed at the available resolution. We expected that some features in the beams of SS 433 would expand and fade. Therefore, MERLIN observed SS 433 exactly simultaneously with the VLBI network, thus enabling visibilities from both networks to be combined in one map, which results in an improved sensitivity for extended low surface-brightness features. Despite scheduling and logistic constraints, we have made six eleven-hour observations at two-day intervals.

Simultaneously, we wanted to track the behaviour of the Doppler shifted lines in the optical spectrum of SS 433, so as to be able to combine the radial and tangential velocity components of the beams, and in order to study correlations between the thermal and nonthermal emission from SS 433. However, the exposure time needed to record the optical spectrum of SS433 is typically only fifteen minutes, and we could therefore aim to study the variability on timescales much shorter than one day. In order to obtain proper twentyfour hour coverage, and to reduce the observing time needed on any particular instrument, we used a total of ten telescopes around the globe. This also minimised the risks of bad weather; for instance, at La Silla, Chile, it was cloudy on ten out of fifteen nights, but our overall program was not substantially damaged.

In support of the spectroscopic observations, multicolour photometry was done. Unfortunately, adverse weather conditions led to a somewhat incomplete coverage.

There were also daily radio flux density measurements at numerous frequencies. This effort started well before the radio mapping, since we wanted to investigate a possible correlation between the occurrence of flares and the generation of bright patches in the beams of SS 433 .

Lastly, the Ginga X-ray satellite observed SS 433 on five consecutive days, at the start of the multifrequency campaign. 


\section{Discussion of the First Results from the 1987 Campaign}

\subsection{RADIO FLUX DENSITY MONITORING}

Measurements at a total of sixteen frequencies, many of them at daily intervals, were obtained by Vermeulen et al. (1989b). They find that SS 433 was in a quiescent state in the last few weeks before the VLBI mapping started, and during the first few days of that central part of the campaign. Then, there was a sequence of flares, in which at least five different events, each lasting several days, can be distinguished. The flaring had not yet ended when monitoring stopped. The authors have found a dip in the total flux density of SS 433 just before the onset of the flaring. They speculate that when flaring starts there is first of all an ejection of some material with large optical depth, such that it blocks some of the steady quiescent emision from SS 433.

After subtracting the quiescent radio emission of SS 433, Vermeulen et al. (1989b) analysed the time and frequency dependence of each of the flares. They conclude that the first event is clearly different from the others. Also, it is clear that these flares are not due to single instantaneous injections of relativistic electrons; rather, processes of sustained generation of relativistic particles are indicated.

\subsection{RADIO SYNTHESIS MAPPING}

Vermeulen et al. (1989c) have obtained a series of six maps at $5 \mathrm{GHz}$, at two-day intervals using Mark III mode A recording with the five-station European VLBI Network (EVN, consisting of Effelsberg, Jodrell Bank, Medicina, Onsala, and the Westerbork tied array). Six hundred and ninety tapes were used, making this the largest experiment to date on the EVN. Additionally, the NRAO $140 \mathrm{ft}$ telescope at Greenbank joined the network for the first, fifth, and sixth of the observations. Also, as was mentioned before, simultaneous MERLIN measurements improved the sensitivity for extended low surface brightness features. However, there were no common stations with the VLBI network, which complicates the relative calibration of the arrays.

The variability of SS 433 leads to difficulties in interpreting the obtained visibilities. As mentioned above, SS 433 was flaring during the measurements; the data of Vermeulen et al. (1989b) indicate flux density changes of up to ten percent during a single eleven-hour synthesis observation. Moreover, the kinematic model predicts that matter in the beams of SS 433 moves by about half a resolution element ( 4.5 mas) during each observation. Work by Vermeulen and Rugers (1989 d) is in progress to develop a hybrid mapping algorithm that takes account of such variability. Despite these problems, the currently available EVN maps already have a higher image fidelity than any previous ones, and they confirm and extend very nicely some of the conclusions of Vermeulen et al. (1987) and Romney et al. (1987).

The radio beams of SS 433 are not continous (jet-like), but rather composed of a series of discrete "blobs" emanating from a core. Note that, by using the difference in proper motion between the approaching (eastern) and receding beam, one can easily identify the central source of the system in a sequence of maps, in contrast to the usual situation for VLBI maps. The locus and proper motion of the blobs are completely consistent with the kinematic model parameters derived from optical observations. In the 1987 series of VLBI maps there is even evidence for the six-day nodding period. Evidently, the motion of these blobs, which emit synchrotron radiation, is the same as that of the thermal matter, which emits Doppler shifted lines. 
Vermeulen et al. (1987) and Fejes et al. (1988) have suggested that there is a radio "brightening zone" at a relatively large distance from SS 433. The flux density evolution of the blobs in the 1987 series of maps is in good agreement with that idea. They brighten for several days after ejection, reaching their maximum flux density at a distance of about $200 \mathrm{AU}$ from the central object, after which they fade rapidly. Work is in progress to verify whether the flux density evolution of the blobs agrees with the suggestion by Vermeulen et al. (1987) that brightening occurs when a blob crosses the bowshock of its predecessor, and with the idea of these authors that the subsequent fading must be ascribed to adiabatic expansion in a region where the passage of previous blobs has created a lower matter density.

The combination of radio maps at one frequency and total flux density measurements at many frequencies yields a powerful diagnostic tool; however, the analysis of this database is as yet far from completed. It is clear that the first of the series of flares discussed in section 3.1 is related to events in or very close to the core of SS 433. Possibly, that first flare occurs when the matter is ejected that later gives rise to the prominent blobs in the VLBI maps. The second flare in the series, on the other hand, reflects the increase in flux density of the blobs in the brightening zone. Indeed, in the map where it is most prominent, the eastern blob alone emits about half of the total flux. This difference in origin between the flares may well explain why they evolve differently, as was discussed in section 3.1. It should be noted that the prominent blobs seen in 1987 are likely to be unusual; in all previous maps of SS 433 the core was the brightest feature. Interestingly, the peak flux density reached by the main "eastern" blob is about three times higher than that of the main "western" one, but this ratio comes closer to unity when account is taken of the expected differential Doppler boosting (Fejes, 1986).

\subsection{OPTICAL SPECTROSCOPY}

The optical spectra collected by Vermeulen et al. (1989e) were obtained on many different instruments; the resolution, spectral range, and integration time vary widely. Therefore, some spectral features can be studied in greater detail than others. The best covered portion of the spectrum contains the blueshifted $\mathrm{H} \alpha$ line; it was observed nearly a hundred times in twenty-one days.

The Doppler shifted lines exhibit variations on a large range of timescales, from several days down to half-an-hour. Episodes of a rather smooth change in wavelength are present, as well as instances of the well-known "bullet-like" behaviour, when a line at one particular wavelength diminishes in strength while a new, separate one rises to prominence at a different wavelength. Up to five such bullets have been seen simultaneously, quite apart from the structure which is often present in the profile of one line.

The wavelength of the shifted lines in general agrees quite well with the predictions of the kinematic model; in the few weeks of the 1987 campaign, the six-day nodding motion shows up especially nicely. When the line profile is complex, it is generally the brightest part which is closest to the predicted wavelength. In the case of multiple lines, the brightest is often the newest one, in agreement with the fast rise to prominence (usually a few hours) and the slower decay (one to several days). However, it should be stressed that the behaviour can vary enormously. During the measurements in 1987 there is a two-day and a three-day period when no Doppler shifted emission lines could be detected at all. Clearly, the beams of SS 433 are fragmented, but models must allow for wide ranges in size, emissivity, and radiative lifetime of the bullets. 
Often, the red- and blueshifted lines behave like mirror images, for example during the switch-off episodes mentioned above. However, there are many instances in the dataset of Vermeulen et al. (1989e) when the redshifted lines change ahead of the blueshifted ones by a few hours or even as much as one day. Moreover, in this dataset the redshifted lines occasionally have a more complex profile than their blueshifted counterparts, but the reverse does not occur. The interpretation of these effects is unclear. Possibly, they find their origin near the compact object, due to some form of partially one-sided ejection. It is perhaps more likely that differences in the local conditions at a greater distance from the binary system are involved, which lead to a dissimilar fragmentation, or to unequal emission measures, or simply to a different obscuration of the two beams.

\subsection{X-RAY MONITORING}

Thanks to a collaborative effort by the Japanese Ginga team and workers at Leicester, $X$-ray data are available for five consecutive days at the start of the 1987 SS 433 monitoring campaign. A preliminary report was presented at the 1988 Dwingeloo workshop on SS 433 by H.C. Pan.

The 1987 observations confirm and extend the earlier EXOSAT work of Watson et al., (1986) and Stewart et al. (1987). The X-ray spectrum of SS 433 can be described by a thermal bremsstrahlung continuum and an Fe XXVII line, at the position predicted by the kinematic model for the blueshifted system. The absence of the redshifted line constrains the length of the X-ray emitting portion of the beams; it also shows that the matter is already fully accelerated at $10^{12} \mathrm{~cm}$ from the compact object.

The Ginga data trace a primary eclipse, in which the compact object is obscured by its companion. The equivalent width of the Doppler shifted line remained constant, indicating that most of the underlying continuum originates in the same region as the emission line (i.e., in the approaching beam). The spectral index softens in the eclipse, which is evidence for a temperature gradient along the X-ray beam.

\section{Summary; Correlations between Different Wavelengths}

There is no clear evidence for a correlation between the evolution seen in the radio maps and the behaviour of the optical Doppler shifted lines. No specific event in the spectra can be linked to the occurrence of the radio flares, and it has thus far not been possible to identify a specific feature on the VLBI maps in which a certain Doppler shifted line originates. That lack of correlation between such widely different emission processes should perhaps not be too surprising. The 1987 campaign has shown that, whereas the beams of SS 433 are clumped on a wide range of scales (minutes to days) in the optical emission lines, the radio emission, in the form of flares or blobs, typically varies on a somewhat larger timescale (days). Thermal material and synchrotron emitting plasma evidently have a different distribution. It is remarkable that they nevertheless both move at $0.26 \mathrm{c}$ according to the predictions of the same kinematic model parameters.

The series of photometric measurements by Aslanov et al. (1989) unfortunately has some gaps during the monitoring campaign. However, these workers do find that SS 433 underwent an optical flare just when the object changed from the radio quiet to the flaring state. Further examination of the shape of the lightcurve during the campaign may reveal whether this simultaneous flare is significant, and, if so, how it should be explained. 
More speculatively, there is a link between the onset of the radio flaring, and the drop in the X-ray flux seen at the very end of the Ginga measurements. This behaviour, if real, would somewhat resemble the suggestion by Band and Grindlay (1986) that when SS 433 is flaring, its X-ray spectrum is (mostly) non-thermal, with a low flux density, but that its spectrum is (partly) thermal, with a higher flux density, when SS 433 is quiescent at radio frequencies.

The 1987 multifrequency campaign has yielded an impressively large set of first class data, thanks to the efforts put in by all the authors of Aslanov et al. (1989) and Vermeulen et al. (1989a to 1989e), by the Japanese Ginga team and the group at Leicester, as well as by other people acknowledged in the references mentioned above. We were fortunate to catch SS 433 in a transition from its quiescent state to the radio flaring state. However, it is not easy to disentangle the emission coming from the central region, near the binary system, from what is being generated further out in the two beams. Perhaps we may conclude that future studies of the radio flaring behaviour of SS433 would not necessarily have to be accompanied by optical spectroscopy, but that such radio studies would again greatly benefit from simultaneous optical photometry and X-ray measurements, given the tantalising hints of correlated behaviour seen in the 1987 campaign.

\section{References}

G.O. Abell, B. Margon; 1979: Nature 279, 701-703

A.A. Aslanov, A.M. Cherepashchuk, V.P. Goranskij, V.Yu. Rahimov,

R.C. Vermeulen; 1989: in preparation

D.L. Band, J.E. Grindlay; 1986: Astrophys. J. 311, 595-606

A.J.B. Downes, T.Pauls, C.J. Salter; 1981: Astron. Astrophys. 103, 277-287

I. Fejes; 1986: Astron. Astrophys. 166, L23-L25

I. Fejes, R.T. Schilizzi, R.C. Vermeulen; 1988: Astron. Astrophys. 189, 124-127

R.M. Hjellming, K.J. Johnston; 1985: in Radio Stars, eds. R.M. Hjellming,

D.M. Gibson, 309-323

B. Margon; 1984: Ann. Rev. Astron. Astrophys. 22, 507-536

J.D. Romney, R.T. Schilizzi, I. Fejes, R.E. Spencer; 1987: Astrophys. J. 321, 822-831

G.C. Stewart, M.G. Watson, M. Matsuoka, W. Brinkmann, J. Jugaku, K. Takagishi,

T. Omodaka, J.C. Kemp, G.D. Henson, D.J. Kraus, T. Mazeh, E.M. Leibowitz; 1987: M.N.R.A.S. 228, 293-303

R.C. Vermeulen, R.T. Schilizzi, V. Icke, I. Fejes, R.E. Spencer; 1987: Nature 328, 309-313

R.C. Vermeulen, P.G. Murdin, P. Angebault, S. D'Odorico, R.T.Schilizzi; 1989a: in preparation

R.C. Vermeulen, W.B. McAdam, S. Trushkin, S. Facondi, R. Fiedler, R. Hjellming,

K. Johnston, J. Corban; 1989 b: in preparation

R.C. Vermeulen, R.T. Schilizzi, R.E. Spencer, J.D. Romney, I. Fejes; 1989c: in preparation

R.C. Vermeulen, M. Rugers; 1989d: in preparation

R.C. Vermeulen, P.G. Murdin, E.P.J. van den Heuvel, S. Fabrika, R.M. Wagner,

B.H. Margon, J.B. Hutchings, R.T. Schilizzi, L.B. van den Hoek, M. van Kerkwijk,

E. Ott, S. D'Odorico; 1989e: in preparation

M.G. Watson, G.C. Stewart, W. Brinkmann, A.R. King; 1986: M.N.R.A.S. 222, 261-271 